\title{
Prevalence of viral HBV and HCV among different group patients in Gujrat Pakistan
}

\author{
Muhammad Irshad ${ }^{1,2}$, Muhammad Naem Raza ${ }^{2}$, Zahid Anwar², Nadia Ikram³, Amber Afroz ${ }^{3}$, \\ Hafiz Muhammad Nasir Iqbal ${ }^{{ }^{*}}$ \\ ${ }^{1}$ Department of Chemistry and Biochemistry, University of Agriculture, Faisalabad, Pakistan \\ ${ }^{2}$ Department of Biochemistry, NSMC University of Gujrat, Gujrat, Pakistan \\ ${ }^{3}$ Department of Biochemistry and Molecular Biology, University of Gujrat, Gujrat, Pakistan \\ Email: ${ }^{*}$ nasir_pk99@hotmail.com
}

Received 6 June 2012; revised 8 July 2012; accepted 27 July 2012

\begin{abstract}
In this study we analyzed blood samples collected from 400 high risk patients for the prevalence of an inflammatory viral disease hepatitis B virus (HBV) and hepatitis C virus (HCV) with the help of standard kit assay and Enzyme-linked immunosorbent assay (ELISA). All the samples were selected randomly from the various places of District Gujrat, Pakistan. All the selected cases were first divided into four groups according to the age and sex (Group 1, Male below age 35 years; Group 2, Male above age 35 years; Group 3, Female below age 35 years; Group 4, Female above age 35 years), each group was comprised of $\mathbf{1 0 0}$ individual patients and analyzed for different parameters for the presence of $\mathrm{HBV}$ and $\mathrm{HCV}$ in comparison with positive and negative controls. The prevalence of $\mathrm{HBV}$ and $\mathrm{HCV}$ was higher in groups 2 $(22 \%)$ and $4(39 \%)$ respectively. Assay profile revealed that the incidence of HCV was higher in female patients as compare to the male patients. The present study indicates that more than $60 \%$ of the cirrhosis and hepatocellular carcinoma in the Region is attributable to hepatitis B virus (HBV) or hepatitis $\mathrm{C}$ virus (HCV) infection.
\end{abstract}

Keywords: HBV; HCV; Liver Inflammation; LFTs; ELISA; ALP; Bilirubin

\section{INTRODUCTION}

Viral hepatitis is frequently fatal and one of commonest liver infectious (inflammatory) disease across the world [1]. Worldwide, probably 2 billion and 350 to 400 million people are chronically infected with this viral infection [2,3]. Approximately 170 million people throughout the world have chronic HCV infection and that 3 - 4 mil-

${ }^{*}$ Corresponding author. lion people are newly infected each year. Hepatitis B and $\mathrm{C}$ virus is present in blood, saliva, semen, vaginal secretions, menstrual blood, and to lesser extent, breast milk, and urine of infected individuals [4]. A highly resilient virus, $\mathrm{HBV}$ or $\mathrm{HCV}$ can easily transmit through contaminated blood transfusion, surgical instruments, dental surgery, sexual contacts and drug abuses, sharing of the house hold items like shaving razors, toothbrushes and also shaving from the barber [4-6].

Patients with slightly elevated ALT (1 - 2 times than the normal limit) are more likely to have transient elevation not because of the disease; however, about $30 \%$ of those with chronic HCV infection have peak ALT. In developing countries where resources and facilities may be significantly limited, the prevalence of $\mathrm{HBV}$ or $\mathrm{HCV}$ is higher as compared to the developed world [7]. In adults daily production of un-conjugated bilirubin is about 250 to $350 \mathrm{mg}$, normal values is $5 \mathrm{mg} / \mathrm{kg} / \mathrm{day}$, or about $400 \mathrm{mg} /$ day. However in comparison to the unconjugated the level of conjugated bilirubin is highly specific for liver disease. The serum bilirubin level is mainly associated with the extent of hepatocellular necrosis. In this regard further studies are needed to characterize the epidemiology of HBV and HCV transmission, especially in Pakistan.

The present study was conducted to investigate the incidence/prevalence of Hepatitis B and C virus in District Gujrat, Pakistan. In this study, we have analyzed a wide range of patients belonging to various health risk groups for the prevalence of $\mathrm{HBV}$ and $\mathrm{HCV}$ infection.

\section{MATERIALS AND METHODS}

\subsection{Sample Collection}

Total 400 clinically diagnosed patients were consecutively recruited for the study from various places of District Gujrat Hospitals, Pakistan. Patients were considered to have hepatitis $\mathrm{B}$ or hepatitis $\mathrm{C}$ infection if their ELIZA 
or screening test was positive and had raised alanineaminotransferase (ALT) level.

\subsection{Blood Samples}

Up to $5 \mathrm{~mL}$ blood was drawn using sterilized disposable syringes from each patient and immediately transferred into the EDTA (anticoagulant) containing tubes. The samples were thoroughly mixed and finally centrifuged at $3000 \mathrm{~g}$ for $5 \mathrm{~min}$. Separated serum fractions were transferred into sterilized eppendorf tubes and stored at $-20^{\circ} \mathrm{C}$ until further use.

\subsection{Determination of Hepatitis B Surface Antigen}

For the purpose of quantitative determination of Hepatitis B surface antigen from a separated serum of all the selected samples, RBP4 (human) ELISA Kit was used in vitro. In this procedure the antigen is "trapped" between two layers of enzyme specific antibodies. The whole reaction is followed by a wash and enzyme activity of the bound material in each Microtiter well is determined by adding the substrate of the enzyme.

\subsection{Liver Function Test (LFT) Profile}

For qualitative detection of $\mathrm{HbsAg}$ in whole blood and serum, hepatitis B surface Antigen (HbsAg) was screened out by the use of Trinity Biotech Uni-Gold HbsAg test kit in vitro. Serum samples were further analyzed for bilirubin (conjugated and unconjugated), and Alkaline phosphatase (ALP).

\subsubsection{Bilirubin}

The total bilirubin in serum and plasma was determined by the method of coupling with diazotized sulfanilic acid $(29 \mathrm{mmol} / \mathrm{L})$ after the addition of caffeine $(130 \mathrm{mmol} / \mathrm{L})$, sodium benzoate $(156 \mathrm{mmol} / \mathrm{L})$ and sodium acetate $(460$ $\mathrm{mmol} / \mathrm{L})$. Absorbance was recorded using spectrophotometer at $578 \mathrm{~nm}$. The direct and indirect bilirubin level was measured according to the method described by Saher et al. [8].

\subsubsection{Alkaline Phosphatase}

To determine the ALP level in the separated serum fractions of all patients, Human Gasellschaft fur Biochemica und Diagnostica mbH (EC 3.1.3.1) kit method was used. Reaction principle is given below:

$$
\begin{gathered}
\text { p-Nitrophenylphosphate }+\mathrm{H}_{2} \mathrm{O} \\
\leftrightarrow \text { phosphate }+ \text { p-nitrophenol }
\end{gathered}
$$

\subsection{Immunochromatographic Test (ICT)}

Immunochromatographic tests strips were obtained from accurate and Acon (Acon, USA). Strips were used ac- cording to the manufacturer's instructions for the purpose of HCV screening in all of the separated serum fractions samples.

\section{RESULTS AND DISCUSSION}

To investigate the prevalence of $\mathrm{HBV}$, all of the selected patients were first screened for the presence of $\mathrm{Hbs} \mathrm{Ag}$ in the sera. The screening assay profile showed that total 31 cases were positive among all of the 400 analyzed samples. In comparison to the HBV the incidence of $\mathrm{HCV}$ was little higher among all groups and total 93 cases were HCV positive from all of the total 400 analyzed samples (Supplementary Table 1-4).

\subsection{Group 1 (Male below Age 35 Years)}

In this group out of the 100 selected samples only $6 \%$ were those having their $\mathrm{HBV}$ positive test. In the same group $14 \%$ patients were highly affected with HCV. Bilirubin assay profile showed that total $18 \%$ patients had the higher Bilirubin level $(1.48 \pm 1.94)$ as compare to the normal value (up to $1.2 \mathrm{mg} / \mathrm{dl}$ ) (Figure 1). While other, $23 \%$ had the higher value of Alakaline phosphtase $(345 \pm$ 18) than that of the normal limits $(80-306 \mathrm{U} / \mathrm{L})$, (Figure 2).

\subsection{Group 2 (Male above Age 35 Years)}

This age group contained $\mathrm{HBV}$ and $\mathrm{HCV}$ positive ratio $10 \%$ and $26 \%$ respectively, out of the total 100 randomly selected samples. Bilirubin test assay revealed that $28 \%$ had the higher level of Bilirubin with maximum of 2.23 $\pm 16 \mathrm{mg} / \mathrm{dl}$ than the normal value while, other $37 \%$ had the higher value of Alakaline phosphtase $(368 \pm 92)$ in comparison to the normal limits (Figures 1-2).

\subsection{Group 3 (Female below Age 35 Years)}

In this group the prevalence of $\mathrm{HBV}$ was $50 \%$ lower as compare to the group 1 (Male below age 35 Years) but

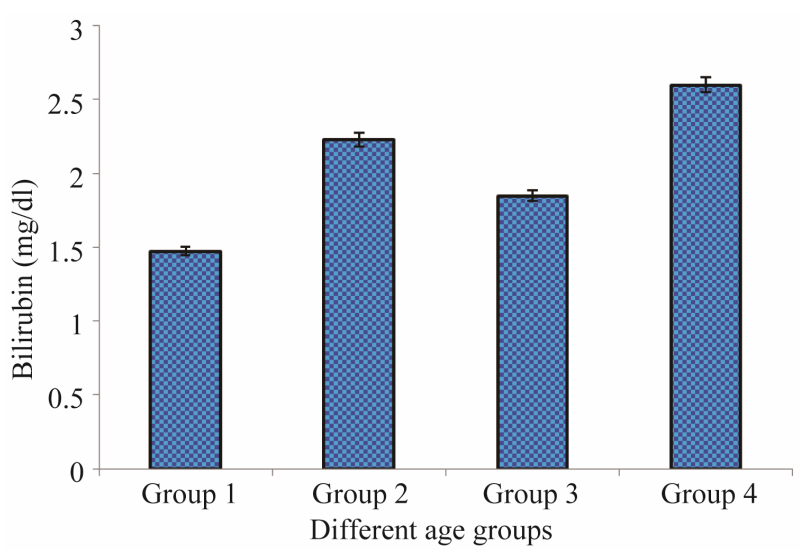

Figure 1. Bilirubin levels in patients of different age groups. 


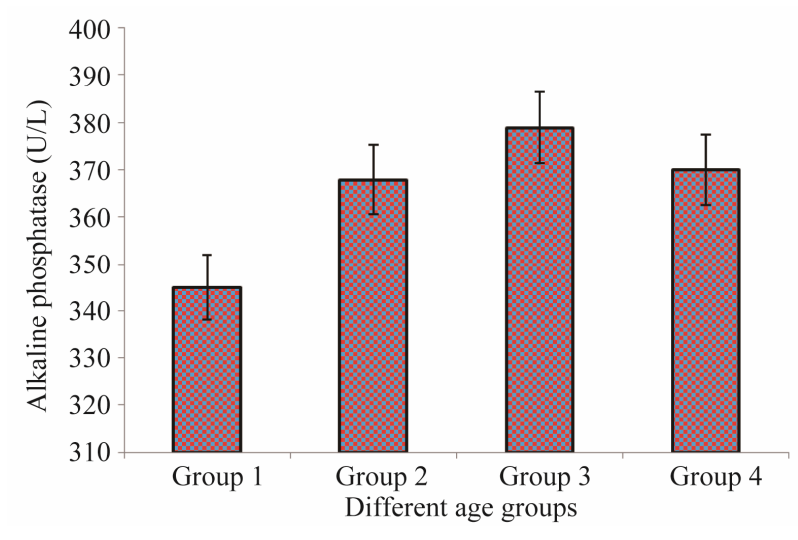

Figure 2. ALP levels in patients of different age groups.

the incidence of $\mathrm{HCV}$ positive cases was surprisingly high in comparison to the same age group of males. Out of 100 patients $22 \%$ had the higher level of Bilirubin $(1.85 \pm 24)$ than that of the normal limits (Figure 1). Total $30 \%$ were found to have higher value of Alakaline phosphtase $(379 \pm 19)$ than its normal limits $(80-306$ U/L) (Figure 2).

\subsection{Group 4 (Female above Age 35 Years)}

In comparison to the all other three groups the patients belong to this group were highly affected with HBV and $\mathrm{HCV}$. Total occurrence of HBV and HCV positive percentage was 12 and $30 \%$ respectively. In this age group $38 \%$ patients have higher level of Bilirubin $(2.6 \pm 11)$ while, $45 \%$ have higher level of Alakaline phosphtase (370 \pm 56$)$ as compare to the normal limits (Figures 12).

Many of the earlier studies from various regions of Pakistan have reported high prevalence of HCV (20.5\% $60 \%),[9,10]$. The poor dental procedures are the major source of HBV or HCV exposure $(39.7 \%)$ that is followed by injections $(16.6 \%)$ and finally the surgical unsterilized items (16.6\%) [11]. In an earlier study, Herve [12] reported that the transmitting risk of these viruses has drastically been reduced by screening for their antibodies before blood transfusion. In the present study we observed higher level of Bilirubin that indicates the biliary inflammation, which may be intrahepatic or extra hepatic and this increased value of Bilirubin, is related to liver hepatitis [13]. In the present study serum ALP values were little higher than many of the previously conducted studies.

Our data suggest that the prevalence of HBV and HCV infection was higher in person with age higher than 35 . Similar findings have also been reported previously by Leung [14]. It has been reported in literature that blood transfusion continues to cause hepatitis $\mathrm{B}$ or $\mathrm{C}$ in those countries, where donor blood is not screened for $\mathrm{HBV}$ [15]. The present study indicates that blood transfusion is taking part in transmission of viral infection. Previously, HBV transmission by blood transfusion has also been reported in Pakistan by [16].

\section{CONCLUSION}

Lack of proper blood screening facilities and unawareness about the possible transmission routes of HBV and $\mathrm{HCV}$ are contributing a great deal towards the spread of the infection among the population. In this regard further studies are needed to characterize the epidemiology of HBV and HCV transmission, especially in Pakistan.

\section{ACKNOWLEDGEMENTS}

The present work was a part of M.Sc. research work conducted by Mr. Muhammad Naem Raza. On providing technical expertise and collaborative help for the present study, Z. Anwar is thankfully acknowledged.

\section{REFERENCES}

[1] Liu, B., Li, J., Han, Y., Liu, Y., Kong, L., Cao, Y. and Huang, Z. (2010) Dynamic analysis of lymphocyte subsets of peripheral blood in patients with acute self-limited hepatitis B. Health, 2, 736-741. doi: $10.4236 /$ health.2010.27112

[2] Noah, D.N., Njouom, R., Bonny, A., Pirsou., Meli, J. and Sida, M.B. (2011) HBs antigene prevalence in blood donors and the risk of transfusion of hepatitis B at the central hospital of yaounde, Cameroon. Open Journal of Gastroenterology, 1, 23-27.

[3] Badar, N., Farooq, U., Ali, S., Nisar, N., Abubakar, M. and Qureshi, J.A. (2012) A molecular approach for genotyping of hepatitis B virus using restriction pattern analysis of samplicon in Pakistan. Open Journal of Medical Microbiology, 2, 16-23. doi:10.4236/ojmm.2012.21003

[4] Lavenchy, D. (2004) Hapetitis B virus epidemiology, disease burden, treatment, and current and emerging prevention and control measures. Journal of Viral Hepatitis, 11, 97-107. doi:10.1046/j.1365-2893.2003.00487.x

[5] Sato, S., Fujiyama, S. and Tanaka, M. (1994) Confection of hepatitis C virus in patients with chronic hepatitis B infection. Journal of Hepatology, 21, 159-166. doi:10.1016/S0168-8278(05)80389-7

[6] Adjei, A.A., Armah, H.B. and Narter-Olaga, E.G. (2006) Seroprevalence of Cytomegalovirus among some voluntary blood donors at the 37 Military Hospital, Accra, Ghana. Ghana Medical Journal, 40, 99-104.

[7] Wild, C.P. and Hall, A.J. (2000) Primary prevention of hepatocellular carcinoma in developing countries. Mutation Research, 462, 381-393. doi:10.1016/S1383-5742(00)00027-2

[8] Saher, F., Rehman, K., Qureeshi, J.A., Irshad, M. and Iqbal, H.M.N. (2012) Investigation of an inflammatory viral disease $\mathrm{HBV}$ in cardiac patients through Polymerase Chain Reaction. Advances in Bioscience and Biotechnology, (Accepted). 
[9] Akhtar, S., Moatter, T., Azam, S.I., Rahbar, M.H. and Adil, S. (1998) Prevalence and risk factors for interfamilial transmission of hepatitis $\mathrm{C}$ virus in Karachi, Pakistan. Baillière's Clinical Haematology, 11, 147-149.

[10] Hamid, H., Iqbal, R., Khan, M.H., Iftikhar, B., Aziz, S., Burki, F.K., Sethi, J., Hassan, M., Nisar, Y.B. and Krishan, J. (2008) Prevalence of hepatitis C in beta thalassaemic major. Gomal Journal of Medical Sciences, 6, 2.

[11] Mohammed, A.J., Qudah, A.A., Shishi, K.F., Sarayreh, A.A. and Quraan, L.A. (2009) Hepatitis C virus (HCV) infection in hemodialysis patients in the south of Jordan. Renal Data Arab World, 20, 488-492.

[12] Herve, P. (2000) Transfusion safety: Emergent or hypothetical risks. Transfusion Clinique et Biologique, 7, 3038 .

[13] Khan, A.A., Parveen, N., Mahaboob, V.S., Rajendraprasad, A., Ravindraprakash, H.R., Venkateswarlu, J.,
Rao, P., Pande, G., Narusu, M.L., Khaja, M.N., Pramila, R., Habeeb, A. and Habibullah, C.M. (2008) Management of hyperbilirubinemia in biliary atresia by hepatic progenitor cell transplantation through hepatic artery: A case report. Transplant Proceedings, 40, 1153-1155. doi:10.1016/j.transproceed.2008.03.110

[14] Leung, N. (2002) Transmission of hepatitis B: Case selection and duration of therapy. Journal of Gastroenterology and Hepatology, 17, 409-414. doi:10.1046/j.1440-1746.2002.02767.x

[15] Fleming (2000) The origin and evolution of hepatitis virus in humans. Journal of General Virology, 82, 693 712.

[16] Rahman, M., Akhtar, G.N. and Lodi, Y. (2002) Transfusion transmitted HIV and HBV infection in Punjab, Pakistan. Pakistan Journal of Medical Science, 18, 18-25. 


\section{Supplementary Data Only for Review}

Table 1. Data of incidence of HBV and HCV in Group 1 (Male below age 35 years).

\begin{tabular}{|c|c|c|c|c|c|c|}
\hline Sr. No. & HBsAg (+ve) & HbsAg $(-v e)$ & Cut off value of HBsAg & $\mathrm{HCV}(+\mathrm{ve})$ & $\mathrm{HCV}(-\mathrm{ve})$ & $\begin{array}{c}\text { Cut off value of } \\
\text { HCV }\end{array}$ \\
\hline 1. & & 0.007 & 0.105 & & 0.016 & 0.150 \\
\hline 2. & & 0.065 & 0.105 & 2.521 & & 0.150 \\
\hline 3. & & 0.009 & 0.105 & & 0.080 & 0.150 \\
\hline 4. & & 0.021 & 0.105 & & 0.005 & 0.150 \\
\hline 5. & & 0.007 & 0.105 & & 0.001 & 0.150 \\
\hline 6. & & 0.012 & 0.105 & & 0.004 & 0.150 \\
\hline 7. & & 0.010 & 0.105 & & 0.017 & 0.150 \\
\hline 8. & & 0.016 & 0.105 & & 0.010 & 0.150 \\
\hline 9. & & 0.018 & 0.105 & & 0.011 & 0.150 \\
\hline 10. & & 0.020 & 0.105 & & 0.033 & 0.150 \\
\hline 11. & & 0.011 & 0.105 & & 0.023 & 0.150 \\
\hline 12. & & 0.010 & 0.105 & & 0.009 & 0.150 \\
\hline 13. & & 0.049 & 0.105 & & 0.021 & 0.150 \\
\hline 14. & & 0.091 & 0.105 & & 0.037 & 0.320 \\
\hline 15. & & 0.014 & 0.105 & 2.569 & & 0.320 \\
\hline 16. & & 0.017 & 0.105 & & 0.021 & 0.320 \\
\hline 17. & & 0.086 & 0.105 & & 0.021 & 0.320 \\
\hline 18. & & 0.021 & 0.105 & & 0.052 & 0.320 \\
\hline 19. & & 0.040 & 0.105 & & 0.078 & 0.320 \\
\hline 20. & & 0.065 & 0.105 & & 0.028 & 0.320 \\
\hline 21. & & 0.041 & 0.105 & 3.222 & & 0.320 \\
\hline 22. & & 0.015 & 0.105 & 2.218 & & 0.320 \\
\hline 23. & & 0.005 & 0.105 & & 0.014 & 0.233 \\
\hline 24. & & 0.019 & 0.105 & 2.451 & & 0.150 \\
\hline 25. & & 0.035 & 0.105 & & 0.010 & 0.150 \\
\hline 26. & & 0.007 & 0.105 & & 0.012 & 0.150 \\
\hline 27. & & 0.014 & 0.105 & & 0.005 & 0.150 \\
\hline 28. & & 0.010 & 0.105 & 0.867 & & 0.150 \\
\hline 29. & & 0.005 & 0.105 & & 0.008 & 0.150 \\
\hline 30. & & 0.012 & 0.105 & & 0.005 & 0.150 \\
\hline 31. & & 0.010 & 0.105 & & 0.007 & 0.150 \\
\hline 32. & & 0.009 & 0.105 & & 0.008 & 0.150 \\
\hline 33. & & 0.010 & 0.105 & & 0.010 & 0.150 \\
\hline 34. & & 0.013 & 0.105 & 3.446 & & 0.150 \\
\hline 35. & & 0.022 & 0.105 & & 0.014 & 0.150 \\
\hline 36. & & 0.016 & 0.105 & & 0.009 & 0.150 \\
\hline 37. & & 0.018 & 0.105 & & 0.014 & 0.150 \\
\hline 38. & & 0.012 & 0.105 & & 0.010 & 0.150 \\
\hline 39. & & 0.013 & 0.105 & 3.786 & & 0.150 \\
\hline 40. & & 0.015 & 0.105 & 1.809 & & 0.150 \\
\hline 41. & & 0.021 & 0.105 & & 0.011 & 0.150 \\
\hline
\end{tabular}




\section{Continued}

\begin{tabular}{|c|c|c|c|c|c|c|}
\hline 42. & & 0.018 & 0.105 & & 0.012 & 0.150 \\
\hline 43. & & 0.011 & 0.105 & 2.294 & & 0.150 \\
\hline 44. & & 0.018 & 0.105 & & 0.016 & 0.150 \\
\hline 45. & & 0.007 & 0.105 & & 0.009 & 0.150 \\
\hline 46. & & 0.025 & 0.105 & & 0.011 & 0.150 \\
\hline 47. & & 0.016 & 0.105 & & 0.074 & 0.150 \\
\hline 48. & & 0.029 & 0.105 & & 0.014 & 0.150 \\
\hline 49. & & 0.010 & 0.105 & & 0.012 & 0.150 \\
\hline 50. & & 0.038 & 0.105 & & 0.009 & 0.150 \\
\hline 51. & & 0.018 & 0.105 & & 0.009 & 0.150 \\
\hline 52. & & 0.009 & 0.105 & & 0.009 & 0.150 \\
\hline 53. & 3.885 & & 0.105 & & 0.013 & 0.150 \\
\hline 54. & & 0.013 & 0.105 & & 0.010 & 0.150 \\
\hline 55. & & 0.013 & 0.105 & & 0.011 & 0.150 \\
\hline 56. & & 0.008 & 0.105 & & 0.008 & 0.150 \\
\hline 57. & & 0.015 & 0.105 & & 0.009 & 0.150 \\
\hline 58. & & 0.016 & 0.105 & & 0.008 & 0.150 \\
\hline 59. & & 0.042 & 0.105 & & 0.015 & 0.150 \\
\hline 60. & & 0.015 & 0.105 & & 0.014 & 0.150 \\
\hline 61. & & 0.008 & 0.105 & & 0.012 & 0.150 \\
\hline 62. & & 0.023 & 0.105 & & 0.017 & 0.150 \\
\hline 63. & & 0.008 & 0.105 & & 0.016 & 0.150 \\
\hline 64. & & 0.021 & 0.105 & & 0.009 & 0.150 \\
\hline 65. & & 0.008 & 0.105 & & 0.010 & 0.150 \\
\hline 66. & & 0.008 & 0.105 & & 0.007 & 0.150 \\
\hline 67. & & 0.008 & 0.105 & & 0.063 & 0.150 \\
\hline 68. & & 0.009 & 0.105 & & 0.006 & 0.150 \\
\hline 69. & & 0.009 & 0.105 & & 0.007 & 0.150 \\
\hline 70. & & 0.007 & 0.105 & & 0.011 & 0.150 \\
\hline 71. & & 0.019 & 0.105 & & 0.012 & 0.150 \\
\hline 72. & & 0.018 & 0.105 & & 0.010 & 0.150 \\
\hline 73. & & 0.024 & 0.105 & & 0.010 & 0.150 \\
\hline 74. & & 0.005 & 0.105 & & 0.006 & 0.150 \\
\hline 75. & & 0.025 & 0.105 & & 0.009 & 0.150 \\
\hline 76. & 3.463 & & 0.105 & & 0.006 & 0.150 \\
\hline 77. & & 0.009 & 0.105 & & 0.007 & 0.150 \\
\hline 78. & & 0.014 & 0.105 & 1.883 & & 0.150 \\
\hline 79. & & 0.013 & 0.105 & & 0.004 & 0.231 \\
\hline 80. & 3.744 & & 0.105 & & 0.039 & 0.231 \\
\hline 81. & & 0.005 & 0.105 & & 0.003 & 0.231 \\
\hline 82. & & 0.009 & 0.105 & & 0.008 & 0.231 \\
\hline 83. & 3.941 & & 0.105 & & 0.006 & 0.231 \\
\hline 84. & & 0.004 & 0.105 & & 0.004 & 0.380 \\
\hline
\end{tabular}




\section{Continued}

\begin{tabular}{|c|c|c|c|c|c|c|}
\hline 85. & & 0.015 & 0.106 & 3.162 & & 0.380 \\
\hline 86. & & 0.046 & 0.106 & & 0.074 & 0.380 \\
\hline 87. & & 0.078 & 0.106 & & 0.086 & 0.380 \\
\hline 88. & & 0.027 & 0.106 & & 0.071 & 0.380 \\
\hline 89. & & 0.031 & 0.106 & & 0.004 & 0.186 \\
\hline 90. & & 0.006 & 0.078 & & 0.003 & 0.186 \\
\hline 91. & & 0.008 & 0.078 & & 0.005 & 0.186 \\
\hline 92. & & 0.009 & 0.078 & 3.077 & & 0.186 \\
\hline 93. & & 0.006 & 0.078 & & 0.003 & 0.186 \\
\hline 94. & & 0.005 & 0.078 & 2.425 & & 0.186 \\
\hline 95. & & 0.055 & 0.233 & & 0.016 & 0.250 \\
\hline 96. & & 0.043 & 0.137 & & 0.011 & 0.250 \\
\hline 97. & 3.629 & & 0.137 & & 0.003 & 0.210 \\
\hline 98. & & 0.020 & 0.088 & & 0.013 & 0.210 \\
\hline 99. & & 0.007 & 0.110 & & 0.008 & 0.210 \\
\hline 100. & 3.763 & & 0.110 & & 0.006 & 0.210 \\
\hline
\end{tabular}

Table 2. Data of incidence of HBV and HCV in Group 2 (Male above age 35 years).

\begin{tabular}{|c|c|c|c|c|c|c|}
\hline Sr. No. & HBsAg $(+v e)$ & HbsAg (-ve) & Cut off value of HBsAg & $\mathrm{HCV}(+\mathrm{ve})$ & $\mathrm{HCV}(-\mathrm{ve})$ & $\begin{array}{c}\text { Cut off value of } \\
\text { HCV }\end{array}$ \\
\hline 1. & & 0.008 & 0.105 & & 0.032 & 0.150 \\
\hline 2. & & 0.037 & 0.105 & 2.998 & & 0.150 \\
\hline 3. & & 0.008 & 0.105 & & 0.008 & 0.150 \\
\hline 4. & & 0.008 & 0.105 & & 0.008 & 0.150 \\
\hline 5. & & 0.034 & 0.105 & & 0.034 & 0.150 \\
\hline 6. & 3.643 & & 0.105 & & 0.019 & 0.150 \\
\hline 7. & & 0.033 & 0.105 & & 0.010 & 0.150 \\
\hline 8. & 3.933 & & 0.105 & & 0.021 & 0.150 \\
\hline 9. & & 0.022 & 0.105 & & 0.016 & 0.150 \\
\hline 10. & & 0.011 & 0.105 & & 0.022 & 0.150 \\
\hline 11. & 3.483 & & 0.105 & & 0.014 & 0.150 \\
\hline 12. & & 0.005 & 0.105 & & 0.009 & 0.150 \\
\hline 13. & & 0.031 & 0.105 & & 0.009 & 0.150 \\
\hline 14. & & 0.052 & 0.105 & & 0.009 & 0.150 \\
\hline 15. & & 0.015 & 0.105 & & 0.020 & 0.150 \\
\hline 16. & 3.394 & & 0.105 & & 0.011 & 0.150 \\
\hline 17. & & 0.013 & 0.105 & 2.840 & & 0.150 \\
\hline 18. & & 0.005 & 0.105 & & 0.007 & 0.150 \\
\hline 19. & 3.741 & & 0.105 & 3.551 & & 0.150 \\
\hline 20. & 1.127 & & 0.105 & & 0.009 & 0.150 \\
\hline 21. & & 0.005 & 0.105 & & 0.007 & 0.150 \\
\hline 22. & & 0.001 & 0.105 & & 0.004 & 0.231 \\
\hline 23. & & 0.005 & 0.105 & & 0.011 & 0.231 \\
\hline 24. & & 0.029 & 0.106 & & 0.008 & 0.231 \\
\hline
\end{tabular}




\section{Continued}

\begin{tabular}{|c|c|c|c|c|c|c|}
\hline 25. & & 0.029 & 0.106 & 3.169 & & 0.380 \\
\hline 26. & & 0.043 & 0.137 & & 0.040 & 0.380 \\
\hline 27. & 3.629 & & 0.137 & & 0.110 & 0.380 \\
\hline 28. & & 0.040 & 0.137 & 3.464 & & 0.250 \\
\hline 29. & & 0.038 & 0.137 & & 0.016 & 0.250 \\
\hline 30. & & 0.038 & 0.080 & & 0.011 & 0.250 \\
\hline 31. & & 0.088 & 0.210 & & 0.003 & 0.250 \\
\hline 32. & & 0.042 & 0.210 & & 0.004 & 0.250 \\
\hline 33. & & 0.039 & 0.210 & & 0.004 & 0.185 \\
\hline 34. & & 0.025 & 0.210 & & 0.030 & 0.210 \\
\hline 35. & & 0.008 & 0.210 & & 0.019 & 0.210 \\
\hline 36. & & 0.015 & 0.210 & 3.646 & & 0.210 \\
\hline 37. & & 0.021 & 0.210 & & 0.004 & 0.210 \\
\hline 38. & & 0.021 & 0.210 & & 0.022 & 0.210 \\
\hline 39. & & 0.023 & 0.190 & 3.469 & & 0.210 \\
\hline 40. & & 0.012 & 0.190 & & 0.007 & 0.210 \\
\hline 41. & & 0.018 & 0.190 & 3.068 & & 0.210 \\
\hline 42. & & 0.017 & 0.105 & 3.060 & & 0.210 \\
\hline 43. & & 0.014 & 0.105 & & 0.052 & 0.210 \\
\hline 44. & & 0.018 & 0.105 & & 0.002 & 0.190 \\
\hline 45. & & 0.023 & 0.105 & & 0.002 & 0.190 \\
\hline 46. & & 0.022 & 0.105 & 2.353 & & 0.190 \\
\hline 47. & & 0.032 & 0.105 & 3.164 & & 0.190 \\
\hline 48. & & 0.022 & 0.105 & & 0.001 & 0.190 \\
\hline 49. & & 0.173 & 0.260 & 3.140 & & 0.190 \\
\hline 50. & & 0.060 & 0.260 & 3.287 & & 0.190 \\
\hline 51. & & 0.063 & 0.260 & & 0.004 & 0.190 \\
\hline 52. & & 0.074 & 0.260 & & 0.004 & 0.190 \\
\hline 53. & 0.249 & & 0.260 & 3.097 & & 0.190 \\
\hline 54. & & 0.220 & 0.260 & & 0.017 & 0.190 \\
\hline 55. & & 0.078 & 0.260 & & 0.003 & 0.190 \\
\hline 56. & & 0.020 & 0.110 & & 0.007 & 0.230 \\
\hline 57. & & 0.013 & 0.110 & & & 0.230 \\
\hline 58. & & 0.016 & 0.110 & 3.964 & & 0.230 \\
\hline 59. & & 0.011 & 0.110 & 3.962 & & 0.230 \\
\hline 60. & & 0.019 & 0.110 & 3.672 & & 0.230 \\
\hline 61. & & 0.009 & 0.110 & & 0.066 & 0.230 \\
\hline 62. & & 0.034 & 0.115 & & 0.024 & 0.230 \\
\hline 63. & & 0.025 & 0.115 & 3.957 & & 0.230 \\
\hline
\end{tabular}




\section{Continued}

\begin{tabular}{|c|c|c|c|c|c|}
\hline 64. & 0.030 & 0.115 & 3.963 & & 0.230 \\
\hline 65. & 0.042 & 0.115 & & 0.021 & 0.230 \\
\hline 66. & 0.040 & 0.115 & & 0.017 & 0.180 \\
\hline 67. & 0.008 & 0.150 & 3.344 & & 0.180 \\
\hline 68. & 0.010 & 0.150 & & 0.004 & 0.180 \\
\hline 69. & 0.018 & 0.150 & 3.208 & & 0.180 \\
\hline 70. & 0.103 & 0.150 & & 0.004 & 0.180 \\
\hline 71. & 0.078 & 0.150 & & 0.008 & 0.180 \\
\hline 72. & 0.022 & 0.107 & & 0.002 & 0.180 \\
\hline 73. & 0.012 & 0.107 & & 0.172 & 0.411 \\
\hline 74. & 0.007 & 0.107 & & 0.139 & 0.411 \\
\hline 75. & 0.051 & 0.107 & & 0.117 & 0.411 \\
\hline 76. & 0.016 & 0.150 & 3.270 & & 0.411 \\
\hline 77. & 0.135 & 0.150 & & 0.003 & 0.411 \\
\hline 78. & 0.017 & 0.150 & & 0.203 & 0.411 \\
\hline 79. & 0.014 & 0.090 & 1.609 & & 0.300 \\
\hline 80. & 0.049 & 0.111 & & 0.003 & 0.300 \\
\hline 81. & 0.057 & 0.111 & 2.974 & & 0.300 \\
\hline 82. & 0.037 & 0.107 & & 0.226 & 0.300 \\
\hline 83. & 0.028 & 0.107 & & 0.009 & 0.300 \\
\hline 84. & 0.025 & 0.099 & & 0.003 & 0.300 \\
\hline 85. & 0.022 & 0.099 & & & 0.300 \\
\hline 86. & 0.025 & 0.099 & & & 0.300 \\
\hline 87. & 0.024 & 0.099 & & & 0.300 \\
\hline 88. & 0.043 & 0.099 & & 0.003 & 0.190 \\
\hline 89. & 0.027 & 0.184 & & 0.004 & 0.190 \\
\hline 90. & 0.020 & 0.082 & & 0.003 & 0.190 \\
\hline 91. & 0.017 & 0.090 & & 0.003 & 0.190 \\
\hline 92. & 0.018 & 0.090 & & 0.003 & 0.250 \\
\hline 93. & 0.019 & 0.090 & & 0.011 & 0.250 \\
\hline 94. & 0.015 & 0.090 & 3.220 & & 0.250 \\
\hline 95. & 0.011 & 0.090 & & 0.004 & 0.190 \\
\hline 96. & 0.022 & 0.106 & & 0.004 & 0.190 \\
\hline 97. & 0.022 & 0.106 & & 0.007 & 0.190 \\
\hline 98. & 0.019 & 0.102 & 2.832 & & 0.193 \\
\hline 99. & 0.008 & 0.102 & & 0.036 & 0.193 \\
\hline 100. & 0.015 & 0.072 & & 0.037 & 0.193 \\
\hline
\end{tabular}


Table 3. Data of incidence of HBV and HCV in Group 3 (Female below age 35 years).

\begin{tabular}{|c|c|c|c|c|c|c|}
\hline Sr. No. & HBsAg $(+v e)$ & HbsAg $(-v e)$ & $\begin{array}{c}\text { Cut off value of } \\
\text { HBsAg }\end{array}$ & $\mathrm{HCV}(+\mathrm{ve})$ & $\mathrm{HCV}(-\mathrm{ve})$ & $\begin{array}{c}\text { Cut off value of } \\
\text { HCV }\end{array}$ \\
\hline 1. & & 0.016 & 0.105 & & 0.037 & 0.150 \\
\hline 2. & & 0.009 & 0.105 & & 0.007 & 0.150 \\
\hline 3. & & 0.041 & 0.105 & & 0.008 & 0.150 \\
\hline 4. & & 0.011 & 0.105 & & 0.009 & 0.150 \\
\hline 5. & & 0.016 & 0.105 & 2.949 & & 0.150 \\
\hline 6. & & 0.027 & 0.105 & & 0.005 & 0.150 \\
\hline 7. & & 0.006 & 0.105 & & 0.019 & 0.150 \\
\hline 8. & & 0.014 & 0.105 & & 0.006 & 0.150 \\
\hline 9. & & 0.009 & 0.105 & & 0.011 & 0.150 \\
\hline 10. & & 0.017 & 0.105 & & 0.023 & 0.150 \\
\hline 11. & & 0.019 & 0.105 & 1.383 & & 0.150 \\
\hline 12. & & 0.012 & 0.105 & & 0.032 & 0.150 \\
\hline 13. & & 0.010 & 0.105 & & 0.011 & 0.150 \\
\hline 14. & & 0.012 & 0.105 & & 0.020 & 0.150 \\
\hline 15. & 0.147 & & 0.105 & & 0.013 & 0.150 \\
\hline 16. & & 0.041 & 0.105 & & 0.015 & 0.150 \\
\hline 17. & & 0.011 & 0.105 & 2.964 & & 0.150 \\
\hline 18. & & 0.018 & 0.105 & 2.675 & & 0.150 \\
\hline 19. & & 0.013 & 0.105 & & 0.013 & 0.150 \\
\hline 20. & & 0.004 & 0.105 & & 0.010 & 0.150 \\
\hline 21. & & 0.004 & 0.105 & & 0.029 & 0.150 \\
\hline 22. & & 0.012 & 0.105 & & 0.039 & 0.320 \\
\hline 23. & & 0.019 & 0.105 & & 0.025 & 0.320 \\
\hline 24. & & 0.040 & 0.105 & & 0.021 & 0.320 \\
\hline 25. & & 0.021 & 0.105 & & 0.037 & 0.320 \\
\hline 26. & & 0.014 & 0.105 & & 0.047 & 0.320 \\
\hline 27. & & 0.030 & 0.105 & & 0.078 & 0.320 \\
\hline 28. & & 0.079 & 0.105 & & 0.014 & 0.320 \\
\hline 29. & & 0.020 & 0.105 & 3.055 & & 0.320 \\
\hline 30. & & 0.011 & 0.105 & 2.900 & & 0.320 \\
\hline 31. & & 0.014 & 0.105 & & 0.063 & 0.320 \\
\hline 32. & & 0.074 & 0.105 & & 0.032 & 0.320 \\
\hline 33. & & 0.047 & 0.105 & & 0.024 & 0.320 \\
\hline 34. & & 0.023 & 0.105 & & 0.020 & 0.320 \\
\hline 35. & & 0.018 & 0.105 & & 0.103 & 0.320 \\
\hline 36. & & 0.016 & 0.105 & & 0.013 & 0.320 \\
\hline 37. & 3.669 & & 0.105 & & 0.069 & 0.320 \\
\hline 38. & & 0.013 & 0.105 & & 0.065 & 0.320 \\
\hline 39. & & 0.031 & 0.105 & & 0.112 & 0.320 \\
\hline 40. & & 0.016 & 0.105 & 1.372 & & 0.320 \\
\hline 41. & & 0.009 & 0.105 & & 0.006 & 0.233 \\
\hline 42. & & 0.069 & 0.105 & & 0.011 & 0.233 \\
\hline
\end{tabular}


Continued

\begin{tabular}{|c|c|c|c|c|c|c|}
\hline 43. & & 0.008 & 0.105 & & 0.014 & 0.233 \\
\hline 44. & & 0.008 & 0.105 & 2.307 & & 0.233 \\
\hline 45. & & 0.015 & 0.105 & & 0.006 & 0.150 \\
\hline 46. & & 0.012 & 0.105 & & 0.021 & 0.150 \\
\hline 47. & & 0.012 & 0.105 & & 0.009 & 0.150 \\
\hline 48. & & 0.014 & 0.105 & & 0.070 & 0.150 \\
\hline 49. & & 0.007 & 0.105 & & 0.051 & 0.150 \\
\hline 50. & & 0.012 & 0.105 & 3.759 & & 0.150 \\
\hline 51. & & 0.010 & 0.105 & 3.417 & & 0.150 \\
\hline 52. & & 0.011 & 0.105 & 1.190 & & 0.150 \\
\hline 53. & & 0.006 & 0.105 & 2.488 & & 0.150 \\
\hline 54. & 3.957 & & 0.105 & & 0.010 & 0.150 \\
\hline 55. & & 0.011 & 0.105 & & 0.006 & 0.150 \\
\hline 56. & & 0.014 & 0.105 & & 0.017 & 0.150 \\
\hline 57. & & 0.016 & 0.105 & 3.007 & & 0.150 \\
\hline 58. & & 0.013 & 0.105 & 1.800 & & 0.150 \\
\hline 59. & & 0.011 & 0.105 & & 0.009 & 0.150 \\
\hline 60. & & 0.009 & 0.105 & 2.567 & & 0.150 \\
\hline 61. & & 0.009 & 0.105 & & 0.005 & 0.150 \\
\hline 62. & & 0.006 & 0.105 & 2.273 & & 0.150 \\
\hline 63. & & 0.013 & 0.105 & & 0.007 & 0.150 \\
\hline 64. & & 0.013 & 0.105 & & 0.004 & 0.150 \\
\hline 65. & & 0.009 & 0.105 & 2.904 & & 0.150 \\
\hline 66. & & 0.016 & 0.105 & & 0.007 & 0.150 \\
\hline 67. & & 0.012 & 0.105 & 1.070 & & 0.150 \\
\hline 68. & & 0.006 & 0.105 & 3.671 & & 0.150 \\
\hline 69. & & 0.013 & 0.105 & & 0.008 & 0.150 \\
\hline 70. & & 0.011 & 0.105 & & 0.023 & 0.150 \\
\hline 71. & & 0.013 & 0.105 & 3.929 & & 0.150 \\
\hline 72. & & 0.024 & 0.105 & 1.176 & & 0.150 \\
\hline 73. & & 0.007 & 0.105 & & 0.009 & 0.150 \\
\hline 74. & & 0.009 & 0.105 & & 0.010 & 0.150 \\
\hline 75. & & 0.039 & 0.105 & & 0.010 & 0.150 \\
\hline 76. & & 0.021 & 0.105 & & 0.010 & 0.150 \\
\hline 77. & & 0.009 & 0.105 & & 0.007 & 0.150 \\
\hline 78. & & 0.018 & 0.105 & 2.608 & & 0.150 \\
\hline 79. & & 0.018 & 0.105 & & 0.009 & 0.150 \\
\hline 80. & & 0.013 & 0.105 & & 0.008 & 0.150 \\
\hline 81. & & 0.015 & 0.105 & & 0.009 & 0.150 \\
\hline 82. & & 0.008 & 0.105 & & 0.009 & 0.150 \\
\hline 83. & & 0.010 & 0.105 & & 0.008 & 0.150 \\
\hline 84. & & 0.008 & 0.105 & 3.565 & & 0.150 \\
\hline 85. & & 0.014 & 0.105 & & 0.013 & 0.150 \\
\hline
\end{tabular}




\section{Continued}

\begin{tabular}{lllll}
\hline 86. & 0.008 & 0.105 & 0.032 & 0.150 \\
87. & 0.013 & 0.105 & 0.007 & 0.150 \\
88. & 0.010 & 0.105 & 0.007 & 0.150 \\
89. & 0.010 & 0.105 & 0.012 & 0.150 \\
90. & 0.007 & 0.105 & 0.008 & 0.150 \\
91. & 0.012 & 0.105 & 0.011 & 0.150 \\
92. & 0.011 & 0.105 & 0.007 & 0.150 \\
93. & 0.011 & 0.105 & 0.017 & 0.150 \\
94. & 0.011 & 0.105 & 0.007 & 0.150 \\
95. & 0.059 & 0.105 & 0.008 & 0.150 \\
96. & 0.005 & 0.105 & 0.005 & 0.150 \\
97. & 0.005 & 0.105 & 0.006 & 0.150 \\
98. & 0.006 & 0.105 & 0.016 & 0.231 \\
99. & 0.006 & 0.105 & 0.191 & 0.231 \\
90. & 0.023 & 0.106 & & 0.231 \\
\hline
\end{tabular}

Table 4. Data of incidence of HBV and HCV in Group 4 (Female above age 35 years).

\begin{tabular}{|c|c|c|c|c|c|c|}
\hline Sr. No. & HBsAg (+ve) & HbsAg $(-v e)$ & $\begin{array}{c}\text { Cut off value of } \\
\text { HBsAg }\end{array}$ & $\mathrm{HCV}(+\mathrm{ve})$ & $\mathrm{HCV}(-\mathrm{ve})$ & $\begin{array}{c}\text { Cut off value of } \\
\text { HCV }\end{array}$ \\
\hline 1. & & 0.004 & 0.105 & & 0.014 & 0.150 \\
\hline 2. & & 0.005 & 0.105 & 2.509 & & 0.150 \\
\hline 3. & & 0.032 & 0.105 & 2.995 & & 0.150 \\
\hline 4. & & 0.013 & 0.105 & & 0.024 & 0.150 \\
\hline 5. & & 0.008 & 0.105 & & 0.028 & 0.150 \\
\hline 6. & & 0.039 & 0.105 & & 0.011 & 0.150 \\
\hline 7. & & 0.013 & 0.105 & & 0.040 & 0.150 \\
\hline 8. & & 0.012 & 0.104 & 3.075 & & 0.190 \\
\hline 9. & & 0.059 & 0.104 & & 0.011 & 0.190 \\
\hline 10. & & 0.033 & 0.104 & & 0.005 & 0.230 \\
\hline 11. & & 0.086 & 0.260 & & 0.026 & 0.230 \\
\hline 12. & & 0.069 & 0.260 & 2.823 & & 0.180 \\
\hline 13. & & 0.011 & 0.110 & 2.571 & & 0.180 \\
\hline 14. & & 0.014 & 0.110 & & 0.117 & 0.180 \\
\hline 15. & & 0.011 & 0.110 & & 0.003 & 0.180 \\
\hline 16. & & 0.033 & 0.115 & 2.436 & & 0.180 \\
\hline 17. & & 0.054 & 0.115 & & 0.007 & 0.180 \\
\hline 18. & & 0.050 & 0.115 & & 0.359 & 0.411 \\
\hline 19. & & 0.011 & 0.150 & 2.783 & & 0.300 \\
\hline 20. & & 0.043 & 0.150 & & 0.066 & 0.300 \\
\hline 21. & & 0.031 & 0.150 & & 0.122 & 0.300 \\
\hline 22. & & 0.126 & 0.150 & & 0.005 & 0.300 \\
\hline 23. & & 0.017 & 0.150 & 2.576 & & 0.300 \\
\hline 24. & & 0.020 & 0.090 & 2.400 & & 0.300 \\
\hline 25. & & 0.015 & 0.090 & 2.258 & & 0.300 \\
\hline
\end{tabular}


Continued

\begin{tabular}{|c|c|c|c|c|c|c|}
\hline 26. & & 0.021 & 0.090 & 2.167 & & 0.190 \\
\hline 27. & & 0.042 & 0.111 & 1.621 & & 0.190 \\
\hline 28. & & 0.044 & 0.111 & & 0.031 & 0.250 \\
\hline 29. & & 0.045 & 0.111 & 2.945 & & 0.190 \\
\hline 30. & 3.496 & & 0.099 & 2.962 & & 0.190 \\
\hline 31. & & 0.019 & 0.099 & 3.070 & & 0.190 \\
\hline 32. & 1.511 & & 0.099 & 3.090 & & 0.190 \\
\hline 33. & & 0.022 & 0.099 & & 0.004 & 0.190 \\
\hline 34. & & 0.017 & 0.184 & & 0.001 & 0.190 \\
\hline 35. & & 0.030 & 0.184 & & 0.016 & 0.190 \\
\hline 36. & & 0.020 & 0.184 & 2.138 & & 0.190 \\
\hline 37. & & 0.022 & 0.184 & & 0.036 & 0.182 \\
\hline 38. & & 0.023 & 0.082 & 2.700 & & 0.182 \\
\hline 39. & & 0.019 & 0.090 & 2.659 & & 0.182 \\
\hline 40. & & 0.023 & 0.090 & 3.221 & & 0.182 \\
\hline 41. & 3.635 & & 0.090 & 2.064 & & 0.182 \\
\hline 42. & & 0.021 & 0.090 & & 0.002 & 0.184 \\
\hline 43. & & 0.026 & 0.090 & & 0.004 & 0.184 \\
\hline 44. & & 0.022 & 0.090 & & 0.006 & 0.184 \\
\hline 45. & & 0.018 & 0.090 & & 0.006 & 0.184 \\
\hline 46. & & 0.011 & 0.090 & 1.662 & & 0.184 \\
\hline 47. & & 0.013 & 0.090 & & 0.007 & 0.185 \\
\hline 48. & & 0.019 & 0.090 & & 0.004 & 0.185 \\
\hline 49. & & 0.025 & 0.106 & & 0.017 & 0.185 \\
\hline 50. & & 0.026 & 0.106 & & 0.012 & 0.185 \\
\hline 51. & & 0.020 & 0.106 & & 0.002 & 0.185 \\
\hline 52. & & 0.002 & 0.063 & & 0.027 & 0.185 \\
\hline 53. & & 0.027 & 0.071 & 3.150 & & 0.184 \\
\hline 54. & & 0.025 & 0.071 & & 0.108 & 0.184 \\
\hline 55. & & 0.008 & 0.071 & 0.436 & & 0.184 \\
\hline 56. & & 0.022 & 0.071 & & 0.132 & 0.184 \\
\hline 57. & & 0.025 & 0.071 & & 0.009 & 0.184 \\
\hline 58. & & 0.024 & 0.071 & 2.850 & & 0.193 \\
\hline 59. & & 0.021 & 0.071 & 2.687 & & 0.193 \\
\hline 60. & & 0.014 & 0.071 & 2.768 & & 0.193 \\
\hline 61. & & 0.005 & 0.071 & 2.780 & & 0.193 \\
\hline 62. & & 0.019 & 0.071 & 2.621 & & 0.195 \\
\hline 63. & & 0.063 & 0.143 & & 0.070 & 0.195 \\
\hline 64. & & 0.076 & 0.143 & 2.465 & & 0.195 \\
\hline 65. & & 0.085 & 0.143 & & 0.005 & 0.184 \\
\hline 66. & & 0.065 & 0.143 & 2.627 & & 0.184 \\
\hline 67. & & 0.055 & 0.143 & & 0.002 & 0.184 \\
\hline 68. & & 0.060 & 0.143 & & 0.010 & 0.184 \\
\hline
\end{tabular}




\section{Continued}

\begin{tabular}{|c|c|c|c|c|c|c|}
\hline 69. & & 0.048 & 0.143 & 2.951 & & 0.184 \\
\hline 70. & & 0.069 & 0.143 & & 0.012 & 0.184 \\
\hline 71. & & 0.064 & 0.143 & & 0.003 & 0.184 \\
\hline 72. & & 0.064 & 0.143 & & 0.003 & 0.184 \\
\hline 73. & & 0.054 & 0.143 & & 0.003 & 0.184 \\
\hline 74. & & 0.052 & 0.143 & 2.855 & & 0.184 \\
\hline 75. & & 0.014 & 0.092 & 2.532 & & 0.184 \\
\hline 76. & & 0.035 & 0.093 & 2.728 & & 0.184 \\
\hline 77. & & 0.016 & 0.093 & & 0.009 & 0.184 \\
\hline 78. & 3.530 & & 0.100 & & 0.006 & 0.184 \\
\hline 79. & & 0.005 & 0.100 & & 0.005 & 0.184 \\
\hline 80. & & 0.016 & 0.100 & 2.516 & & 0.184 \\
\hline 81. & & 0.007 & 0.100 & 2.709 & & 0.184 \\
\hline 82. & & 0.012 & 0.100 & & 0.005 & 0.184 \\
\hline 83. & & 0.017 & 0.106 & 2.981 & & 0.184 \\
\hline 84. & & 0.010 & 0.083 & & 0.006 & 0.184 \\
\hline 85. & & 0.010 & 0.103 & & 0.004 & 0.184 \\
\hline 86. & & & 0.102 & & 0.004 & 0.184 \\
\hline 87. & 2.938 & 0.043 & 0.072 & 3.178 & & 0.184 \\
\hline 88. & & & & & 0.007 & 0.184 \\
\hline 89. & & & & & 0.003 & 0.184 \\
\hline 90. & 3.741 & & & 2.082 & & 0.189 \\
\hline 91. & 1.127 & & & 2.109 & & 0.189 \\
\hline 92. & 3.741 & & & 2.104 & & 0.189 \\
\hline 93. & & & & & 0.010 & 0.189 \\
\hline 94. & 1.127 & & & 0.534 & & 0.184 \\
\hline 95. & 2.222 & & & 2.702 & & 0.184 \\
\hline 96. & & & & 2.854 & & 0.184 \\
\hline 97. & 3.741 & & & 2.774 & & 0.184 \\
\hline 98. & & & & & 0.007 & 0.192 \\
\hline 99. & & & & & 0.005 & 0.192 \\
\hline 100. & 1.127 & & & 3.115 & & 0.192 \\
\hline
\end{tabular}

